\title{
Editorial: Microbial Production of Biopolyesters and Their Building Blocks: Opportunities and Challenges
}

\author{
Huibin Zou ${ }^{1,2 *}$, Seiichi Taguchi ${ }^{3}$ and David Bernard Levin ${ }^{4}$ \\ ${ }^{1}$ College of Chemical Engineering, Qingdao University of Science and Technology, Qingdao, China, ${ }^{2}$ CAS Key Laboratory of Bio- \\ based Materials, Qingdao Institute of Bioenergy and Bioprocess Technology, Chinese Academy of Sciences, Qingdao, China, \\ ${ }^{3}$ Faculty of Life Sciences and Agriculture, Tokyo University of Agriculture, Tokyo, Japan, ${ }^{4}$ Department of Biosystems Engineering, \\ Faculty of Agricultural and Food Sciences, University of Manitoba, Winnipeg, MB, Canada
}

Keywords: biopolyesters, microbial biotechnology, poly(lactic acid), polyhydroxyalkanoates, biomonomers

\section{Editorial on the Research Topic}

Microbial Production of Biopolyesters and their Building Blocks: Opportunities and Challenges

Although polyhydroxyalkanoates were discovered over 100 hundred years ago, and have been used for many applications (Choi et al., 2020), the majority of global polyester supply still relies on traditional fossil-feedstocks, with well-developed chemical techniques and significant advantages in economy and scale. The barriers for the microbial production of biopolyesters major include: 1) high production and downstream purification costs (Wang et al., 2019); 2) limited production capacity and supply of non-food bio-feedstocks; and 3) limited diversity of monomers and final products to adapt to marketing requirements (Zheng et al., 2020).

To solve these bottleneck barriers, large members of research institutes and industries have contributed their endeavors in this developing field. Other than polyhydroxyalkanoates, semisynthesized polylactic acid (PLA) has become another bulk commercialized biopolyester with acceptable unit cost and promising material properties (Castro-Aguirre et al., 2016). Other novel biopolyesters are also in fast development. For example, "unnatural" lactate containing polyesters can be microbially produced after systematic engineering of key enzymes and chassis strains (Taguchi et al., 2008; Choi et al., 2016). After that, new 2-hydroxy monomeric constitutes such as glycolate and 2-hydroxybutyrate could be incorporated into the polymeric backbone (Taguchi and Matsumoto, 2020). Furthermore, novel aromatic biopolyesters like d-phenyl lactate containing polyesters can also be produced by engineered strains (Yang et al., 2018).

Moreover, the diversity of bio-monomers has increased in recent years (Taguchi and Matsumoto, 2020). In addition to well-known bio-organic acids (lactic acid and succinic acid), bio-diols like 1,4butanediol and 1,3-propanediol, have achieved high-titer production using engineered strains (Yim et al., 2011; Ju et al., 2020). These bio-monomers can be utilized as drop-in chemicals in the production of commercial polyesters like poly(butylene succinate) (PBS), poly(butylene terephthalate) (PBT), and poly(propylene terephthalate) (PPT).

In this Research Topic, a number of experts contributed their updated research outcomes or opinions regarding the strategies to improve the microbial production of polyester. From the perspective of microbial production of novel lactate containing polyesters and oligomers, Nduko and Taguchi have provided insights into the history for the development of lactate containing polyesters/oligomers, and the applications of variable lactate containing polyesters/oligomers (as macromonomer building blocks). Relating to the use of non-food feedstocks in the bioproduction of polyesters, Sun et al. and Moriya et al. present their updated studies on the microbial production of poly(3-hydroxybutyrate) from a broader-range of non-food substrates. Relating to the microbial production of medium chain-length 
polyhydroxyalkanoates, Scheel et al. reported a fermentation protocol which can be applied to control the copolymer composition to exhibit increased flexibility and elasticity in a series of medium chain-length poly(3-hydroxyalkanoates). Harada et al. present a study on the microbial production of medium chain-length poly(3-hydroxyalkanoates) by application of an engineered polyhydroxyalkanoate synthase, which significantly improved the 3-hydroxyhexanoate $(3 \mathrm{HHx})$ fraction in the copolymers. Dartiailh et al. studied the effects of monomer composition on the thermal and mechanical properties of medium chain-length polyhydroxyalkanoate synthesized by Pseudomonas putida cultured with different substrates.

It is hard to forecast the future of polyester industries, but we believe biopolyesters will have a promising future, based on: the availability of renewable and sustainable bio-feedstocks

\section{REFERENCES}

Castro-Aguirre, E., Iñiguez-Franco, F., Samsudin, H., Fang, X., and Auras, R. (2016). Poly(lactic Acid)-Mass Production, Processing, Industrial Applications, and End of Life. Adv. Drug Deliv. Rev. 107, 333-366. doi:10.1016/ j.addr.2016.03.010

Choi, S. Y., Park, S. J., Kim, W. J., Yang, J. E., Lee, H., Shin, J., et al. (2016). One-step Fermentative Production of Poly(lactate-Co-Glycolate) from Carbohydrates in Escherichia coli. Nat. Biotechnol. 34, 435-440. doi:10.1038/nbt.3485

Choi, S. Y., Rhie, M. N., Kim, H. T., Joo, J. C., Cho, I. J., Son, J., et al. (2020). Metabolic Engineering for the Synthesis of Polyesters: A 100-year Journey from Polyhydroxyalkanoates to Non-natural Microbial Polyesters. Metab. Eng. 58, 47-81. doi:10.1016/j.ymben.2019.05.009

Ju, J.-H., Wang, D., Heo, S.-Y., Kim, M.-S., Seo, J.-W., Kim, Y.-M., et al. (2020). Enhancement of 1,3-propanediol Production from Industrial By-Product by Lactobacillus Reuteri CH53. Microb. Cell Fact. 19, 6. doi:10.1186/s12934-0191275-x

Taguchi, S., and Matsumoto, K. i. (2020). Evolution of Polyhydroxyalkanoate Synthesizing Systems toward a Sustainable Plastic Industry. Polym. J. 53, 67-79. doi:10.1038/s41428-020-00420-8

Taguchi, S., Yamada, M., Matsumoto, K., Tajima, K., Satoh, Y., Munekata, M., et al. (2008). A Microbial Factory for Lactate-Based Polyesters Using a LactatePolymerizing Enzyme. Proc. Natl. Acad. Sci. 105, 17323-17327. doi:10.1073/ pnas.0805653105

Wang, Y., Ling, C., Chen, Y., Jiang, X., and Chen, G.-Q. (2019). Microbial Engineering for Easy Downstream Processing. Biotechnol. Adv. 37, 107365. doi:10.1016/j.biotechadv.2019.03.004 to support the bioproduction of polyesters; consumer demand for biodegradable and bioassimilatable materials based on renewable biopolymers; industries and research institutes that are eager to develop renewable and degradable polyester products; and the increasing application of advanced biotechnologies (like synthetic biology with the goal of making novel bio-monomers) and materials science techniques (like the research on biomaterials made from functional biopolyesters) that are being applied in this field.

Enjoy reading!

\section{AUTHOR CONTRIBUTIONS}

$\mathrm{HZ}$, ST, and DL conceived the manuscript; HZ wrote the manuscript; ST and DL reviewed and edited the manuscript.

Yang, J. E., Park, S. J., Kim, W. J., Kim, H. J., Kim, B. J., Lee, H., et al. (2018). Onestep Fermentative Production of Aromatic Polyesters from Glucose by Metabolically Engineered Escherichia coli Strains. Nat. Commun. 9, 1-10. doi:10.1038/s41467-017-02498-w

Yim, H., Haselbeck, R., Niu, W., Pujol-Baxley, C., Burgard, A., Boldt, J., et al. (2011). Metabolic Engineering of Escherichia coli for Direct Production of 1,4butanediol. Nat. Chem. Biol. 7, 445-452. doi:10.1038/NCHEMBIO.580

Zheng, Y., Chen, J.-C., Ma, Y.-M., and Chen, G.-Q. (2020). Engineering Biosynthesis of Polyhydroxyalkanoates (PHA) for Diversity and Cost Reduction. Metab. Eng. 58, 82-93. doi:10.1016/j.ymben.2019.07.004

Conflict of Interest: The authors declare that the research was conducted in the absence of any commercial or financial relationships that could be construed as a potential conflict of interest.

Publisher's Note: All claims expressed in this article are solely those of the authors and do not necessarily represent those of their affiliated organizations, or those of the publisher, the editors and the reviewers. Any product that may be evaluated in this article, or claim that may be made by its manufacturer, is not guaranteed or endorsed by the publisher.

Copyright (c) 2021 Zou, Taguchi and Levin. This is an open-access article distributed under the terms of the Creative Commons Attribution License (CC BY). The use, distribution or reproduction in other forums is permitted, provided the original author(s) and the copyright owner(s) are credited and that the original publication in this journal is cited, in accordance with accepted academic practice. No use, distribution or reproduction is permitted which does not comply with these terms. 